Having thus ascertained the law which (to the best of our information) appears to govern the influence of climate upon European life in India, we are justified in inferring that the same law operates in other countries. Indeed the conclusion is inevitable until it shall have been shewn, in any particular instance, that some other law prevails. Our requirements, therefore, for determining the true law of mortality among Europeans residing abroad are limited to the determination of the values of the single constant representing the extra risk for the respective climates. And herein lies the great importance of the discovery of general laws, viz., that by their aid we are enabled to economize our facts, and, by bringing them all to bear upon a single point, compel them, as it were, to yield us information which we should otherwise be unable to obtain. As an instance of this I may refer to the Tables published in Vol. 7, p 134, of this Journal, embodying a considerable collection of facts relating to foreign risks. These facts, although insufficient to serve for the construction of independent Tables of mortality for different climates, are yet numerous enough to enable us to determine, with a tolerable degree of accuracy, the single constant required for the adjustment of the Home lable to each case; and I may perhaps on some future occasion ask the indulgence of your readers for an analysis of the experience referred to.

I am, Sir,

Your very obedient servant,

$$
\begin{array}{r}
\text { 10, King Street, Cheapside, } \\
\text { 27th February, } 1868 .
\end{array}
$$

W. M. MAKEHAM.

\title{
DEMONSTRATION OF A FORMULA FOR INTERPOLATION.
}

\section{To the Editor of the Assurance Magazine.}

Sir,-I have been asked on more than one occasion how the formula were obtained which I used in graduating the mortality among the males of the peerage (Assurance Magazine, vol. xii., p. 221); and as the question may not be without interest to some readers of the Journal, I will now state the method.

I had noticed the following resemblance between two sets of expressions. If a series $u_{0}, u_{5}, u_{10}$, \&c. is differenced, and $\Delta u_{0}, \Delta^{2} u_{0}, \ldots$ be the initial terms of each order of differences; $\Delta^{4} u_{0}$ being constant; then, as is shown on page 23 of the current volume of the Journal,

$$
\begin{array}{rlrl}
\delta u_{0} & =\frac{1}{5} \Delta u_{0}-\frac{2}{5^{2}} \Delta^{2} u_{0}+\frac{6}{5^{3}} \Delta^{3} u_{0}-\frac{21}{5^{4}} \Delta^{4} u_{0} \\
\delta^{2} u_{0} & = & \frac{1}{5^{2}} \Delta^{2} u_{0}-\frac{4}{5^{3}} \Delta^{3} u_{0}+\frac{16}{5^{4}} \Delta^{4} u_{0} \\
\delta^{3} u_{0}= & \frac{1}{5^{3}} \Delta^{3} u_{0}-\frac{6}{5^{4}} \Delta^{4} u_{0} \\
\delta^{4} u_{0}= & \frac{1}{5^{4}} \Delta^{4} u_{0}
\end{array}
$$


Also, if $\Sigma_{1}$ be the sum of the first five terms $u_{0}+\ldots+u_{4}$ of a series, $\Sigma_{2}$ the sum of the next five terms $u_{5}$ to $u_{9}$, and so on; and $\Delta \Sigma_{1}, \Delta^{2} \Sigma_{1}$, $\Delta^{3} \Sigma_{1}$, the differences of these sums, their third difference being coustant instead of the fourth, it may be proved by subtracting from one another the values of $\Sigma_{1}, \Sigma_{2}$, \&c. in terms of $u_{0}, \delta u_{0}, \delta^{2} u_{0}$, \&c. that

$$
\begin{array}{rlrl}
\delta u_{0} & =\frac{1}{5^{2}} \Delta \Sigma_{1}-\frac{4}{5^{3}} \Delta^{2} \Sigma_{1}+\frac{16}{5^{4}} \Delta^{3} \Sigma_{1} \\
\delta^{2} u_{0} & =\frac{1}{5^{3}} \Delta^{2} \Sigma_{1}-\frac{6}{5^{4}} \Delta^{3} \Sigma_{1} \\
\delta^{3} u_{0} & = & \frac{1}{5^{4}} \Delta^{3} \Sigma_{1}
\end{array}
$$

For according to the ordinary formula for the sum of any number of terms of a series,

$$
\begin{gathered}
\Sigma_{1}=5 u_{0}+\frac{5.4}{1.2} \delta u_{0}+\frac{5.43}{1.2 .3} \delta^{2} u_{0}+\frac{5.4 .3 .2}{1.2 .3 .4} \delta^{3} u_{0} \\
\Sigma_{2}=10 u_{0}+\frac{10.9}{1.2} \delta u_{0}+\frac{10.9 .8}{1.2 .3} \delta^{2} u_{0}+\frac{10.9 .8 .7}{1.2 .3 .4} \delta^{3} u_{0}-\Sigma_{1} \\
\Sigma_{3}=15 u_{0}+\frac{15 \cdot 14}{1.2} \delta u_{0}+\frac{15.14 .13}{1.2 .3} \delta^{2} u_{0}+\frac{15.14 .13 .12}{1.2 .3 .4} \delta^{3} u_{0}-\left(\Sigma_{1}+\Sigma_{2}\right) \\
\Sigma_{4}=20 u_{0}+\frac{20.19}{1.2} \delta u_{0}+\frac{20.19 .18}{1.2 .3} \delta^{2} u_{0}+\frac{20.19 .18 .17}{1.2 .3} \cdot \frac{1}{3} \delta^{3} u_{0}-\left(\Sigma_{1}+\Sigma_{2}+\Sigma_{3}\right) \\
\therefore \Sigma_{1}=5 u_{0}+10 \delta u_{0}+10 \delta^{2} u_{0}+5 \delta^{3} u_{0} \\
\Sigma_{2}=5 u_{0}+35 \delta u_{0}+110 \delta^{2} u_{0}+205 \delta^{3} u_{0} \\
\Sigma_{3}=5 u_{0}+60 \delta u_{0}+335 \delta^{2} u_{0}+1155 \delta^{3} u_{0} \\
\Sigma_{4}=5 u_{0}+85 \delta u_{0}+685 \delta^{2} u_{0}+3480 \delta^{3} u_{0}
\end{gathered}
$$

If these quantities are differenced, the first column of differences will be

$$
\begin{aligned}
& 25 \delta u_{0}+100 \delta^{2} u_{0}+200 \delta^{3} u_{0}=\Delta \mathrm{Y}_{1} \\
& 25 \delta u_{0}+225 \delta^{2} u_{0}+950 \delta^{3} u_{0} \\
& 25 \delta u_{0}+350 \delta^{2} u_{0}+2325 \delta^{3} u_{0}
\end{aligned}
$$

the second column

$$
\begin{aligned}
& 125 \delta^{2} u_{0}+750 \delta^{3} u_{0}=\Delta^{2} \Sigma_{1} \\
& 125 \delta^{2} u_{0}+1375 \delta^{3} u_{0}
\end{aligned}
$$

and the last difference

$$
\begin{gathered}
625 \delta^{3} u_{0}=\Delta^{3} \Sigma_{1} \\
\therefore \delta^{3} u_{0}=\frac{1}{5^{4}} \Delta^{3} \Sigma_{1} \text {, as stated in (7). }
\end{gathered}
$$

Substituting this value of $\delta^{3} u_{0}$ in (9) we have

$$
\begin{aligned}
\Delta^{2} \Sigma_{1} & =125 \delta^{2} u_{0}+\frac{750}{5^{4}} \Delta^{3} \Sigma_{1} \\
\therefore \delta^{2} u_{0} & =\frac{1}{5^{3}} \Delta^{2} \Sigma_{1}-\frac{6}{5^{4}} \Delta^{3} \Sigma_{1}
\end{aligned}
$$


and substituting these values of $\delta^{2} u_{0}$ and $\delta^{3} u_{0}$ in (8) we have

$$
\begin{aligned}
\Delta \Sigma_{1} & =25 \delta u_{0}+\frac{100}{5^{3}} \Delta^{2} \Sigma_{1}-\frac{600}{5^{4}} \Delta^{3} \Sigma_{1}+\frac{200}{5^{4}} \Delta^{3} \Sigma_{1} \\
& =25 \delta u_{0}+\frac{100}{5^{3}} \Delta^{2} \Sigma_{1}-\frac{400}{5^{4}} \Delta^{3} \Sigma_{1} \\
\therefore \delta u_{0} & =\frac{1}{5^{2}} \Delta \Sigma_{1}-\frac{4}{5^{3}} \Delta^{2} \Sigma_{1}+\frac{16}{5^{4}} \Delta^{3} \Sigma_{1}
\end{aligned}
$$

Now it will be seen that the coefficients of $\Delta \Sigma_{1}, \Delta^{2} \Sigma_{1}$, \&c. in (5) are the same as those of $\Delta^{2} u_{0}, \Delta^{3} u_{0}, \& c$. in (2); and the same identity of coefficients occurs in (3) and (6) and in (4) and (7). As this identity could not be accidental I proceeded to try substitution of coefficients on a series involving a larger number of differences. The method of obtaining $\delta u_{0}$, \&c. from the differences $\left(\Delta u_{0}, \Delta^{2} u_{0}, \& c\right.$. $)$ of every $h$ th term of the original series is given by $\mathrm{Mr}$. Neison in his Contributions to Vital Statistics, and by means of it $I$ found the values of $\delta^{2} u_{0}, \delta^{3} u_{0}$, \&c. in terms of $\Delta^{2} u_{0}, \Delta^{3} u_{0}$, \&c., putting $h=10$, and supposing the sixth difference constant; the value of $\delta u_{0}$ not being required. These are as follows:-

$$
\begin{aligned}
\delta^{2} u_{0} & =\frac{1}{10^{2}} \Delta^{2} u_{0}-\frac{9}{10^{3}} \Delta^{3} u_{0}+\frac{77 \cdot 25}{10^{4}} \Delta^{4} u_{0}-\frac{669 \cdot 75}{10^{5}} \Delta^{5} u_{0}+\frac{5895 \cdot 225}{10^{6}} \Delta^{6} u_{0} \\
\delta^{3} u_{0} & = \\
\delta^{4} u_{0} & = \\
\frac{1}{10^{3}} \Delta^{3} u_{0}-\frac{13 \cdot 5}{10^{4}} \Delta^{4} u_{0}+\frac{146 \cdot 25}{10^{5}} \Delta^{5} u_{0}-\frac{1480 \cdot 5}{10^{6}} \Delta^{6} u_{0} & \frac{1}{10^{4}} \Delta^{4} u_{0}-\frac{18}{10^{5}} \Delta^{5} u_{0}+\frac{235 \cdot 5}{10^{6}} \Delta^{6} u_{0} \\
\delta^{5} u_{0}= & \frac{1}{10^{5}} \Delta^{5} u_{0}-\frac{22}{10^{6}} \Delta^{6} u_{0} \\
\delta^{6} u_{0}= & \frac{1}{10^{6}} \Delta^{6} u_{0}
\end{aligned}
$$

Then reducing the indices of $\delta^{2} u_{0}, \delta^{3} u_{0}$, \&c., and of $\Delta^{2} u_{0}, \Delta^{3} u_{0}$, \&c. by
unity, and substituting $\Sigma_{1}$ for $u_{0}$, these being the changes necessary to convert the formulæ (2), (3) and (4) into (5), (6) and (7), I arrived at the following formulx, which proved correct in the nsing.

$$
\begin{array}{lcc}
\delta^{1} u_{0}=\cdot 01 \Delta \Sigma_{1}-009 \Delta^{2} \Sigma_{1}+\cdot 007725 \Delta^{3} \Sigma_{1}-\cdot 0066975 \Delta^{4} \Sigma_{1}+\cdot 005895225 \Delta^{5} \Sigma_{1} \\
\delta^{2} u_{0}= & .001 \Delta^{2} \Sigma_{1}-\cdot 00135 \Delta^{3} \Sigma_{1}+\cdot 0014625 \Delta^{4} \Sigma_{1}-\cdot 0014805 \Delta^{5} \Sigma_{1} \\
\delta^{3} u_{0}= & \cdot 0001 \Delta^{3} \Sigma_{1}-\cdot 00018 \Delta^{4} \Sigma_{1}+\cdot 0002355 \Delta^{5} \Sigma_{1} \\
\delta^{4} u_{0}= & \cdot 00001 \Delta^{4} \Sigma_{1}-\cdot 0000225 \Delta^{5} \Sigma_{1} \\
\delta^{5} u_{0}= & & \cdot 000001 \Delta^{5} \Sigma_{1}
\end{array}
$$

In vol. xii, page 221 , however, the $u_{0}$ and $\Sigma_{1}$ are omitted: $\delta$ stands there for $\delta u_{0}$ and $\Delta$ for $\Delta \Sigma_{1}$.

\section{1, Fleet Street, 4th March, 1868.}

Yours obediently, 